\title{
Healthcare resource consumption and cost of care among patients with polycystic kidney disease in Italy
}

\section{Luca Degli Esposti' \\ Chiara Veronesi' \\ Valentina Perrone' \\ Stefano Buda' \\ Antonio Santoro ${ }^{2}$}

'Clicon S.r.l. Health, Economics \& Outcomes Research, Ravenna, Italy; 2Department of Nephrology and Dialysis, Policlinico S. Orsola-Malpighi, Bologna, Italy
Correspondence: Luca Degli Esposti Clicon S.r.l. Health, Economics \& Outcomes Research, Via salara 36, 48100 Ravenna, Italy

Tel +3954438393

Fax +39544212699

Email luca.degliesposti@clicon.it
This article was published in the following Dove Press journal:

ClinicoEconomics and Outcomes Research

27 April 2017

Number of times this article has been viewed

Objective: The aim of this study was to assess healthcare resource consumption and its associated costs among patients with polycystic kidney disease (PKD) in a real-world setting.

Methods: An observational retrospective cohort analysis was conducted using data from the administrative databases of four Italian local health units. Data for patients who were diagnosed with PKD during the inclusion period (January 1, 2010 to December 31, 2012) were extracted. The date on which a patient's first PKD hospitalization occurred during the inclusion period was defined as the index date (ID), and the ID was defined as the date of the first dialysis treatment recorded during the inclusion period for patients undergoing dialysis. Data regarding the clinical characteristics of patients included in the study during the 12 months prior to the ID (pre-ID; characterization period) were collected. All patients were then followed up for the 12 months following the ID (post-ID; follow-up period). Healthcare consumption and its associated costs were analyzed during the follow-up period. All costs are reported in euros (€).

Results: A total of 1,123 patients with PKD were included in this study, $61.9 \%$ of whom were male; the mean age of the patients was $57.7 \pm 24.5$ years. At diagnosis, $11.2 \%$ and $1.1 \%$ of patients were affected by the dominant and recessive forms of PKD, respectively. Approximately $8 \%$ of the included patients were undergoing dialysis at ID (baseline). The incidence of dialysis was fourfold greater among patients with autosomal-dominant PKD (ADPKD) than among the total cohort (33.3\% compared with an overall 8.3\%). During the follow-up period, the average annual rates of healthcare resource consumption were greater among dialyzed than non-dialyzed patients. The average healthcare expenditures were $€ 45,059.62$ and $€ 3,913.89(p<0.001)$ per year for dialyzed and non-dialyzed PDK patients, respectively. Our findings suggest that in the real-world Italian context, consumption of healthcare among patients with PKD has increased at dialysis initiation due to the cost of outpatient specialist healthcare services as well as other costs. Research on the prevention of PKD-related complications and disease progression may help to facilitate a decrease in the costs associated with this condition.

Keywords: polycystic kidney disease; autosomal-dominant polycystic kidney disease; autosomal-recessive polycystic kidney disease; real-world setting

\section{Introduction}

Polycystic kidney disease (PKD) is an inherited disorder characterized by the appearance of cysts in both kidneys, leading to progressive renal failure. There are two main types of PKD that are caused by different genetic mutations: the autosomal-dominant form (ADPKD, autosomal-dominant PKD) is observed most frequently and often identified in late onset cases, and the recessive form (ARPKD, autosomal-recessive 
PKD) is observed less frequently but is often lethal when identified during the first month of life.

ADPKD is best known as "adult's polycystic disease" because its symptoms and signs are identified only during adulthood. This form is more common than the form identified during childhood and affects $\sim 12.5$ million people worldwide. The distribution of ADPKD does not differ by sex or race. ${ }^{1,2}$ The prevalence of ADPKD is often overestimated, but recent epidemiological reports suggest that this condition has an incidence lower than $4: 10,0000^{3,4}$

Among individuals with this condition, the growth and expansion of cysts in both kidneys lead to renal failure, and approximately half of affected patients require kidney dialysis or a kidney transplant by the age of 54 years..$^{5-8}$ ADPKD is the fourth leading cause of kidney failure, and patients affected by this condition account for $\sim 10 \%$ of renal replacement cases. ${ }^{9}$

As a result of medical advances, ADPKD is often diagnosed more frequently, and the implementation of strategies for the early diagnosis and treatment of renal and extra-renal complications has improved both quality of life and life expectancy among ADPKD patients. ${ }^{1,10,11}$

The natural history of this disease has been found to be associated with significant and substantial economic burden on the health system. Estimates suggest that the annual direct healthcare costs associated with ADPKD amount to $€ 2$ billion in Europe alone. ${ }^{1}$

The aim of this study was to assess healthcare resource consumption and analyze its associated costs among patients with PKD in a real-world setting.

\section{Methods}

\section{Data sources}

This analysis was performed using data from the administrative databases (DBs) of four Italian local health units (LHUs) located in Campania, Lombardia, Lazio, and Sicilia, with a total patient population of $\sim 3.6$ million health-assisted individuals.

The structure of these DBs, which are routinely updated for administrative and reimbursement purposes, has been described in detail elsewhere. ${ }^{12}$ The following DBs were used in this study: the beneficiaries DB, which contains patient demographic data; the medication prescription DB, which comprises information, including the AnatomicalTherapeutic-Chemical code, number of packages ordered, number of units per package, dosages, unit cost per package, and prescription date for each drug purchased for the patient; the hospital discharge DB, which contains data regarding all hospitalizations and includes the discharge diagnosis codes (classified according to the International Classification of Diseases, Ninth Revision, Clinical Modification [ICD-9-CM]) and diagnosis-related group (DRG) reimbursement rate; and the ambulatory care specialist $\mathrm{DB}$, which includes records for all outpatient specialist services provided to the patient. The DBs were linked via anonymized patient codes. Informed consent is not required for using encrypted retrospective information, according to Italian regulations. In accordance with the AIFA Determination, ${ }^{13}$ Guidelines for classification and conduction of observational studies on drugs, this study was notified to the local ethics committee in each participating LHU according to the Italian law regarding the conduct of observational analysis, and the LHU ethics committees approved the study.

\section{Cohort definition}

This observational retrospective study included all patients aged $\geq 18$ years and identified as having at least one hospitalization with a primary or secondary diagnosis of PKD (ICD-9-CM code: 753.1) between the January 1, 2010, and December 31, 2012 (inclusion period). The date of the first PKD-associated hospitalization occurring during the inclusion period was defined as the index date (ID) for non-dialyzed patients; for patients on dialysis, the ID was defined as the date of the first recorded dialysis treatment during the inclusion period. The clinical characteristics of included patients during the 12-month period prior to the ID were collected (characterization period). All patients were then followed up for 12 months following the ID (follow-up period). "Dialyzed patients" were defined as those who had at least one record indicating the receipt of outpatient specialist services related to dialysis (procedure codes 38.95, 39.95, 54.93, 54.98, and 97.29.1) during or after the ID. During the inclusion period, patients were also classified according to the diagnosis received upon discharge from $\mathrm{PKD}$ hospitalization as suffering from ADPKD, ARPKD, or an unspecified PKD genotype using the following ICD-9-CM codes: 753.13, 753.14 , and 753.12, respectively. Patients who did not have any of the ICD-9-CM codes listed above were categorized as "other".

Healthcare consumption was defined by the sum of the costs associated with the prescribed treatments, outpatient specialist services, and hospital admission identified during the follow-up period. Patients transferred to other LHUs during the follow-up period were excluded from this analysis. 


\section{Cost analysis}

The mean annual healthcare costs per patient were evaluated based on total resource consumption (in term of drugs, hospitalizations, and outpatient specialist services) during the follow-up period. A cost analysis was performed according to the Italian National Health System (NHS) guidelines. All costs are reported in euros $(€)$. Drug costs were evaluated at Italian NHS purchase prices. Hospitalization costs were determined using the DRG tariffs, which represent the NHS reimbursement levels received by healthcare providers. The costs of instrumental and laboratory tests were determined according to the tariffs applied by the evaluated regions.

\section{Statistical analysis}

Continuous variables are reported as means and standard deviations (mean $\pm \mathrm{SD}$ ), whereas categorical variables are expressed as numbers and percentages. Differences were considered statistically significant at a $p$ value of $\leq 0.05$.

In cases in which data were not available due to privacy concerns, where results referred to less than four patients ${ }^{14}$ (potentially identifiable data), results are presented as NI (not issuable). All analyses were performed using STATA software version 12.1 (Stata Corp. LP, College Station, TX).

\section{Results}

The records for a total of 1,123 patients PKD were included in the study; 695 of the enrolled patients $(\sim 62 \%$ of a total sample) were males. At baseline, patients on dialysis accounted for $\sim 8 \%$ of the total population. Table 1 summarizes the baseline demographic and clinical characteristics of the study population stratified according to dialysis status.

At baseline, $11.2 \%(n=126)$ and $1.1 \%(n=13)$ of patients were affected by the dominant and recessive forms of PKD, respectively (Table 1). Of the included patients, $16.6 \%$ $(n=186)$ had an unspecified genotype, and the remaining patients were identified as having uncharacterized PKD ( $\mathrm{n}=798,71.1 \%$ of the total sample).

The four subpopulations of PKD patients differed slightly in terms of their baseline demographic and clinical characteristics. The gender distribution within each subpopulation of PKD patients was relatively equal. The mean $( \pm$ SD) age of the 1,123 patients included in the analysis at the ID was $57.7 \pm 24.5$ years. The mean $( \pm \mathrm{SD})$ age of the patients with the recessive form of PKD was $41.6 \pm 35.5$ years. Patients in this cohort were younger than those in the other PKD subpopulations. Patients suffering from ADPKD were, on average, younger than those in the non-ADPKD group; the mean age of patients in the ADPKD group was 48.3 years, whereas the mean age of patients in the sample overall was 57.7 years, and the mean age of patients in the group of patients for whom a PKD diagnosis was unspecified was slightly higher (61.0 years).

While patients suffering from ADPKD were, on average, younger than patients in the non-ADPKD group, the

Table I Baseline demographic and clinical characteristics of the study population stratified according to PKD subtype and dialysis status at the index date

\begin{tabular}{llll}
\hline & Non-dialyzed (n, \%) & Dialyzed (n, \%) & Total (n, \%) \\
\hline All patients with PKD diagnosis & $1,030(91.7)$ & $93(8.3)$ & $1,123(100)$ \\
Age (mean \pm SD) & $57.5 \pm 25.2$ & $60.1 \pm 14.8$ & $57.7 \pm 24.5$ \\
Male & $631(61.3)$ & $31(62.9)$ & $695(61.9)$ \\
Autosomal dominant & $84(66.7)$ & $42(33.3)$ & $126(100.0)$ \\
Age (mean \pm SD) & $43.1 \pm 24.7$ & $58.8 \pm 13.6$ & $48.3 \pm 22.8$ \\
Male & $52(61.9)$ & $24(57.1)$ & $76(60.3)$ \\
Autosomal recessive & $12(92.3)$ & $\mathrm{NI}$ & $13(100.0)$ \\
Age (mean \pm SD) & $37.8 \pm 35.9$ & - & $41.6 \pm 35.5$ \\
Male & $7(58.3)$ & $\mathrm{NI}$ & $8(61.5)$ \\
Genotype unspecified & $159(85.5)$ & $27(14.5)$ & $186(100.0)$ \\
Age (mean \pm SD) & $61.4 \pm 24.9$ & $58.9 \pm 13.3$ & $61.0 \pm 23.5$ \\
Male & $94(59.1)$ & $20(74.1)$ & $114(61.3)$ \\
Other & $775(97.1)$ & $23(2.9)$ & $798(100.0)$ \\
Age (mean $\pm S D)$ & $58.6 \pm 24.5$ & $62.9 \pm 19.0$ & $58.7 \pm 24.4$ \\
Male & $478(61.7)$ & $19(83.0)$ & $497(62.3)$ \\
\hline
\end{tabular}

Notes: All included patients were classified as "dialyzed patients" based on the presence of at least one recording of outpatient specialist services related to dialysis (codes $38.95,39.95,54.93,54.98$, and 97.29.1) during or after the index date. Patients were also classified according to the discharge diagnosis, after hospitalization for PKD, as suffering from ADPKD, ARPKD, or unspecified genotype if they had the ICD-9-CM codes: 753.13, 753.14, and 753.12, respectively. Patients who did not have any of the ICD-9-CM codes listed above are defined as "other."

Abbreviations: ADPKD, autosomal-dominant polycystic kidney disease; ARPKD, autosomal-recessive polycystic kidney disease; ICD-9-CM, International Classification of Diseases, Ninth Revision, Clinical Modification; PKD, polycystic kidney disease; SD, standard deviation; NI, not issuable. 
Table 2 Healthcare consumption (in terms of the average annual number of pharmaceutical prescriptions, ambulatory care services, and hospital admissions) during the follow-up period stratified according to PKD subtype

\begin{tabular}{|c|c|c|c|c|c|c|c|c|}
\hline & \multicolumn{2}{|c|}{ Autosomal dominant } & \multicolumn{2}{|c|}{ Autosomal recessive } & \multicolumn{2}{|c|}{ Genotype unspecified } & \multicolumn{2}{|l|}{ Other } \\
\hline & Mean & \pm SD & Mean & $\pm \mathbf{S D}$ & Mean & $\overline{ \pm \text { SD }}$ & Mean & $\pm \mathbf{S D}$ \\
\hline \multicolumn{9}{|c|}{ Pharmaceutical prescriptions } \\
\hline Non-dialyzed & 14.5 & 16.0 & 10.1 & 10.1 & 16.6 & 14.8 & I5.I & 14.0 \\
\hline Dialyzed & 27.7 & 15.3 & $\mathrm{NI}$ & - & 31.6 & 20.4 & 29.0 & 16.8 \\
\hline Total & 18.8 & 16.9 & 13.1 & 13.0 & 18.8 & 16.6 & 15.5 & 14.3 \\
\hline \multicolumn{9}{|c|}{ Ambulatory care services } \\
\hline Non-dialyzed & 5.1 & II.I & 2.0 & 3.7 & 6.6 & 8.7 & 5.1 & 6.7 \\
\hline Dialyzed & 28.2 & 13.2 & $\mathrm{NI}$ & - & 29.8 & 13.9 & 26.7 & 14.2 \\
\hline Total & 12.7 & 16.1 & 4.7 & 8.7 & 10.0 & 12.6 & 5.7 & 7.9 \\
\hline \multicolumn{9}{|c|}{ Hospital admissions } \\
\hline Non-dialyzed & 0.8 & 1.3 & 1.4 & 1.8 & 0.9 & 1.5 & 0.8 & 1.3 \\
\hline Dialyzed & 1.2 & 1.7 & $\mathrm{NI}$ & - & 1.6 & 1.2 & 1.6 & 1.5 \\
\hline Total & 0.9 & 1.4 & 1.2 & 1.7 & 1.0 & 1.5 & 0.9 & 1.3 \\
\hline
\end{tabular}

Notes: All included patients were classified as "dialyzed patients" based on the presence of at least one recording of outpatient specialist services related to dialysis (codes 38.95, 39.95, 54.93, 54.98, and 97.29.1) during or after the index date. Patients were also classified according to the discharge diagnosis, after hospitalization for PKD, as suffering from ADPKD, ARPKD, or unspecified genotype if they had the ICD-9-CM codes: 753.13, 753.14, and 753.12, respectively. Patients who did not have any of the ICD-9-CM codes listed above are defined as "other".

Abbreviations: ADPKD, autosomal-dominant polycystic kidney disease; ARPKD, autosomal-recessive polycystic kidney disease; ICD-9-CM, International Classification of Diseases, Ninth Revision, Clinical Modification; PKD, polycystic kidney disease; SD, standard deviations; NI, not issuable.

incidence of dialysis at the ID was four times greater among ADPKD patients than that identified within the sample overall (33.3\% compared with an overall 8.3\%). During the 1-year follow-up period, $\sim 1.3 \%$ of patients underwent kidney transplantation.

The following analysis of healthcare consumption and its associated costs related focused only on the cohort of patients with confirmed genotypes, as the small sample of ARPKD patients included in this study may limit the generalizability of the derived results.

Healthcare consumption (in terms of average annual number of pharmaceutical prescriptions, ambulatory care services, and hospital admission) during the follow-up period is described according to dialysis and ADPKD status in Table 2. During the study period, healthcare resource consumption was higher in the ADPKD dialyzed-patient group than in non-dialyzed patient group. The rates of healthcare resource consumption were 27.7 and 14.5 for drug prescriptions and 0.8 and 1.2 for hospital admission among individuals in the dialyzed and non-dialyzed ADPKD groups, respectively, and the average rate of ambulatory care services utilization was five times higher among dialyzed ADPKD patients than their non-dialyzed counterparts (28.2 compared to 5.1).

The annual healthcare costs associated with the management of PKD following the ID are reported in Figure 1. The average cost of healthcare during the 1-year follow-up period was higher among dialyzed than non-dialyzed PKD patients $(p<0.001)$. Specifically, the mean annual cost per patient in the dialyzed group was $€ 45,059.62$ (of which $€ 34,840.62$, $€ 6,298.85$, and $€ 3,920.15$ were attributable to ambulatory care, hospitalization, and pharmaceutical consumption, respectively); however, among non-dialyzed PKD patients, the average annual cost of care was $€ 3,913.89$ (of which $€ 985.83$, €2,445.46, and $€ 482.6$ were attributable to drug treatments, hospitalization, and ambulatory care services, respectively).

The mean annual healthcare costs per patients based on resource consumption from ID and by PKD subtype are shown in Figure 2. During the follow-up period, the mean annual costs of healthcare were $€ 45,390$ and $€ 4,287$ $(p<0.001)$ among ADPKD patients undergoing dialysis and per patient not undergoing dialysis, respectively.

\section{Discussion}

PKD is one of the most common genetic disorders worldwide and represents the most frequently observed genetic cause of end-stage renal disease in adults. ${ }^{15,7}$

Few data regarding healthcare resource consumption in patients with PKD are currently available. ${ }^{16-19}$ Nevertheless, studies have shown that patients with ADPKD incur healthcare costs throughout their lifespan due to outpatient care and hospitalizations..$^{20}$ The currently available evidence suggests that medical costs double with progression of chronic kidney disease (CKD) to a higher stage. ${ }^{21,22}$ Indeed, healthcare resource utilization and costs associated with PKD increase steeply when a patient reaches advanced stages of the disease and when the patient requires dialysis or transplantation..$^{21,23}$

In accordance with the results of previous studies, ${ }^{16-18}$ this retrospective analysis, conducted in a "real-world" setting, shows that the percentage of patients with ADPKD who 


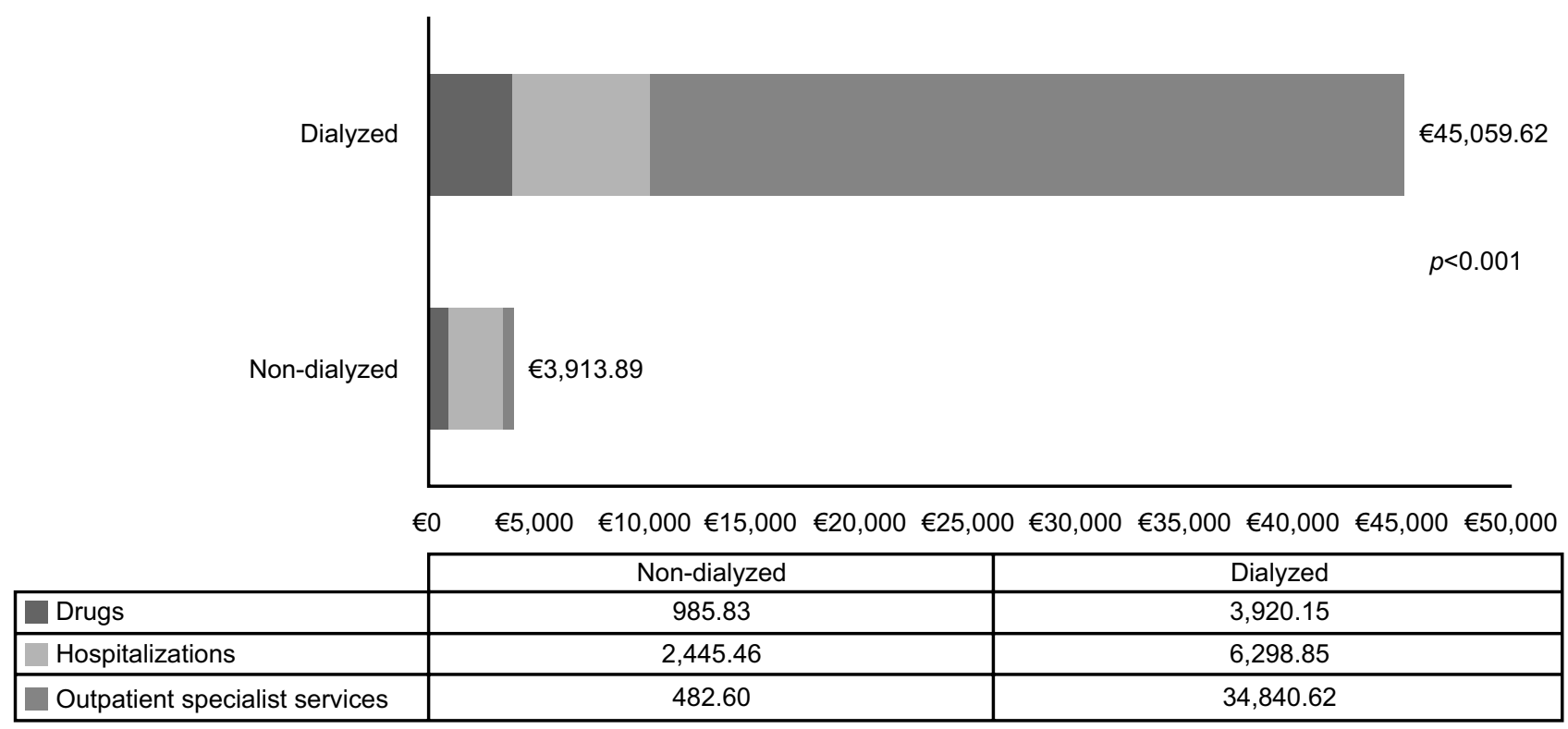

Figure I Mean annual cost per PKD patient during the follow-up period. Costs are reported in euros $(€)$.

Notes: All included patients were classified as "dialyzed patients" based on the presence of at least one record of outpatient specialist services related to dialysis (codes 38.95, 39.95, 54.93, 54.98, and 97.29.1) during or after the index date. Patients were also classified according to the discharge diagnosis, after hospitalization for PKD, as suffering from ADPKD, ARPKD, or unspecified genotype if they had the ICD-9-CM codes: 753.13, 753.14, and 753.12, respectively. Patients who did not have any of the ICD-9-CM codes listed above are defined as "other."

Abbreviation: ADPKD, autosomal-dominant polycystic kidney disease; ARPKD, autosomal-recessive polycystic kidney disease; ICD-9-CM, International Classification of Diseases, Ninth Revision, Clinical Modification; PKD, polycystic kidney disease.

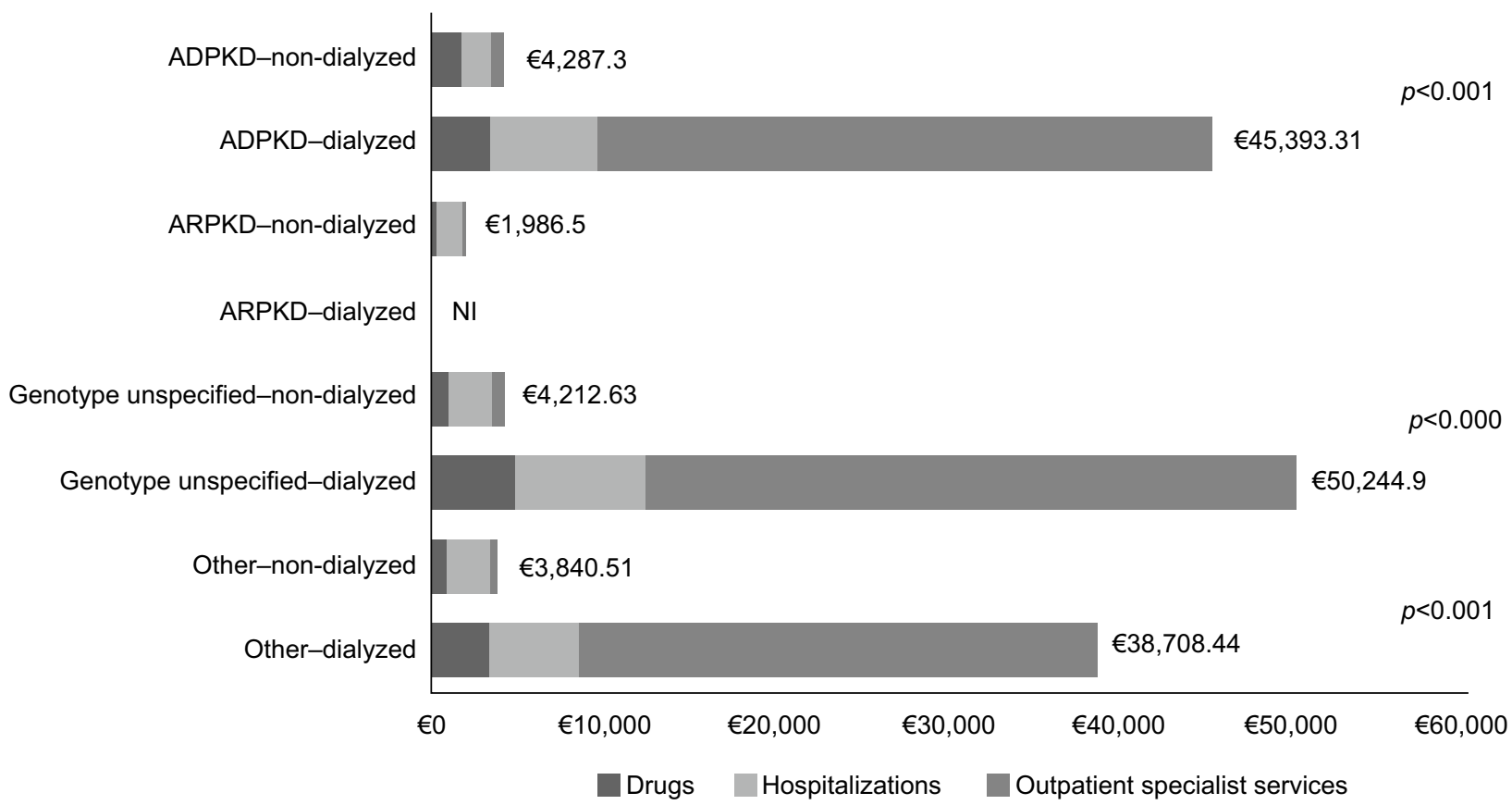

Figure 2 Mean annual cost per patient during the follow-up period stratified according to PKD subtypes (ADPKD, ARPKD, unspecified genotype, or other). Costs are reported in euros $(€)$.

Notes: All included patients were classified as "dialyzed patients" based on the presence of at least one record of outpatient specialist services related to dialysis (codes 38.95, 39.95, 54.93, 54.98, and 97.29.1) during or after the index date. Patients were also classified according to the discharge diagnosis, after hospitalization for PKD, as suffering from ADPKD, ARPKD, or unspecified genotype if they had the ICD-9-CM codes: 753.13, 753.14, and 753.12, respectively. Patients who did not have any of the ICD-9-CM codes listed above are defined as "other."

Abbreviations: ADPKD, autosomal-dominant polycystic kidney disease; ARPKD, autosomal-recessive polycystic kidney disease; ICD-9-CM, International Classification of Diseases, Ninth Revision, Clinical Modification; NI, not issuable. 
required dialysis was about four times greater when compared with the proportion of the overall population requiring dialysis treatment. In addition, the annual healthcare resources consumption identified among ADPKD patients on dialysis was higher than that observed among non-dialysis patients.

A recent US study reported that $20 \%$ of patients with early-stage CKD require at least one hospitalization every 6 months after ADPKD diagnosis, and that this percentage increases to $51 \%$ and $74 \%$ in post-transplant and dialysis patients, respectively. ${ }^{18}$

The recent "Position paper for the prevention of CKD," which was approved during the State-Regional Conference on August 8, 2014, suggested that delaying progression to renal failure by least 5 years for $10 \%$ of subjects with stage III to stage IV CKD and delaying the onset of dialysis for at least 5 years would result in savings of up to $€ 2.5$ billion annually. ${ }^{24}$ As estimated by this document, the annual direct cost of treating a hemodialysis patient is approximately $€ 43,800.00$. The most recent data provided by the European Society of Nephrology Registry, Dialysis and Transplantation (ERA-EDTA) suggest that the cost associated with dialysis and transplantation among European ADPKD patients is estimated at $\sim € 1.5$ billion (of which the estimated annual cost per each hemodialysis patient amounts to $€ 55,500) .{ }^{9}$ However, these estimates do not provide a comprehensive picture of the economic burden associated with the disease, as they do not account for the expenses incurred by patients who did not undergo any dialysis treatments or transplantation, those associated with the treatment of complications (e.g., infections), or the indirect costs resulting from lost productivity.

In accordance with previous studies, our cost analysis suggested that the mean annual healthcare expenditure was higher in the group of patients who underwent dialysis $(\sim € 45,060)$ than in those who did not undergo dialysis $(\sim € 3,914)$. In consideration of the significant difference observed in the costs of outpatient specialist healthcare services between dialyzed and non-dialyzed patients, these data suggest that dialysis may be the main driver of costs for the NHS. This finding is particularly interesting, given that the subpopulation of patients with ADPKD had an incidence of dialysis that was four times higher than that observed in the entire study population.

Our study has many limitations that should be considered during the interpretation of our results. These limitations include the following. First, given the observational nature of this study and its basis on data collected through DBs, we cannot exclude the possibility that some data may have been missed due to inaccurate or lost medical records. Second, because we used administrative DBs, we lacked information on some relevant clinical information that may serve as a specific indicator of disease severity; and third, we analyzed a relatively limited sample size of patients. In fact, we focused the majority of our analysis only on one cohort of patients with genetically confirmed ADPKD, as the small sample of ARPKD patients may have limited the generalizability of these data. Thus, large studies are needed to confirm our results in different patient populations.

\section{Conclusion}

Although further studies including larger patient samples are needed to confirm these results, the cost of adult PKD has a substantial impact on the healthcare system throughout the entire disease course. Our findings suggest that the consumption of healthcare among Italian patients with PKD increased at dialysis initiation due to the cost of outpatient specialist healthcare services as well as other costs. Research on the prevention of PKD-related complications and disease progression may contribute to controlling the costs associated with this condition.

\section{Acknowledgments}

Manuscript development was supported by unconditional funding from Otsuka.

The abstract of this paper was presented at the ISPOR 19th Annual European Congress as a poster presentation. The poster's abstract was published in "Poster Abstracts" Volume 19, Value in Health journal in November 2016, DOI: http:// dx.doi.org/10.1016/j.jval.2016.09.993.

\section{Disclosure}

The authors report no conflicts of interest in this work.

\section{References}

1. Chapman AB, Devuyst O, Eckardt KU, et al. Autosomal-dominant polycystic kidney disease (ADPKD): executive summary from a Kidney Disease: improving Global Outcomes (KDIGO) Controversies Conference. Kidney Int. 2015;88(1):17-27.

2. Torres VE, Harris PC, Pirson Y. Autosomal dominant polycystic kidney disease. Lancet. 2007;369(9569):1287-1301.

3. Willey CJ, Blais JD, Hall AK, Krasa HB, Makin AJ, Czerwiec FS. Prevalence of autosomal dominant polycystic kidney disease in the European Union. Nephrol Dial Transplant. Epub 2016 Jun 19.

4. Blanchette CM, Matter S, Chawla AJ, Gutierrez B. Burden of autosomal dominant polycystic kidney disease: Systematic literature review. $\mathrm{Am}$ J Pharm Benefits. 2015;7(2):e27-e36.

5. Takiar V, Caplan MJ. Polycystic kidney disease: pathogenesis and potential therapies. Biochim Biophys Acta. 2011;1812(10):1337-1343.

6. Alam A, Perrone RD. Management of ESRD in patients with autosomal dominant polycystic kidney disease. Adv Chronic Kidney Dis. 2010;17:164-172.

7. Igarashi P, Somlo S. Genetics and pathogenesis of polycystic kidney disease. J Am Soc Nephrol. 2002;13(9):2384-2398.

8. Grantham JJ. Autosomal dominant polycystic kidney disease. $N$ Engl J Med. 2008;359:1477-1485. 
9. Spithoven EM, Kramer A, Meijer E, et al. Renal replacement therapy for autosomal dominant polycystic kidney disease (ADPKD) in Europe: prevalence and survival-an analysis of data from the ERA-EDTA Registry. Nephrol Dial Transplant. 2014;29(Suppl 4):iv15-iv25.

10. Ong AC, Devuyst O, Knebelmann B, Walz G; ERA-EDTA Working Group for Inherited Kidney Diseases. Autosomal dominant polycystic kidney disease: the changing face of clinical management. Lancet. 2015;385(9981):1993-2002.

11. Kühn WE, Walz G. The treatment of autosomal dominant polycystic kidney disease. Dtsch Ärztebl Int. 2015;112(51-52):884-890.

12. Perrone V, Sangiorgi D, Buda S, Degli Esposti L. Disease progression and health care resource consumption in patients affected by hepatitis C virus in real practice setting. Clinoecon Outcomes Res. 2016;8: 591-597.

13. AIFA Guideline for the classification and conduction of the observational studies on medicines. Available from: https://www.agenziafarmaco.gov. it/ricclin/sites/default/files/files_wysiwyg/files/CIRCULARS/Circular \%2031st\%20May\%202010.pdf. Accessed February 20, 2017.

14. Opinion $05 / 2014$ on Privacy and Data Protection. Available from: http:// ec.europa.eu/justice/data-protection/article-29/documentation/opinionrecommendation/files/2014/wp216_it.pdf. Accessed February 20, 2017.

15. Woon C, Bielinski-Bradbury A, O'Reilly K, Robinson P. A systematic review of the predictors of disease progression in patients with autosomal dominant polycystic kidney disease. BMC Nephrol. 2015;16:140.

16. McEwan P, Bennett H, O’Reilly K, Robinson P. Quantifying the impact of treatment on the public health burden of adpkd: a Uk case study using the adpkd outcomes model. Value Health. 18(7):A763.
17. Blanchette CM, Craver C, Belk KW, Lubeck DP, Rossetti S, Gutierrez B. Hospital-based inpatient resource utilization associated with autosomal dominant polycystic kidney disease in the US. J Med Econ. 2015;18(4):303-311.

18. Knight TG, Schaefer C, Krasa H, Oberdhan D, Chapman A, Perrone R. Economic burden of autosomal dominant polycystic kidney disease in the United States (Abstract 160). Am J Kidney Dis. 2013;61(4):A55.

19. Eriksson D, Karlsson L, Eklund O, et al. Autosomal polycystic kidney disease (Adpkd): costs and resource utilisation in the Nordic Countries. Value Health. 2015;18(7):A513.

20. European ADPKD Forum, gennaio 2015. Tradurre le conoscenze in strategie politiche per migliorare la cura della malattia policistica renale autosomica dominante in Europa. Available from: http://www.pkdinternational.org/eaf_adpkd_forum_policy_report_2015/Accessed May 13, 2016.

21. Knight T, Schaefer C, Krasa H, Oberdhan D, Chapman A, Perrone RD. Medical resource utilization and costs associated with autosomal dominant polycystic kidney disease in the USA: a retrospective matched cohort analysis of private insurer data. Clincoecon Outcomes Res. 2015;7:123-132.

22. Honeycutt AA, Segel JE, Zhuo X, Hoerger TJ, Imai K, Williams D. Medical costs of CKD in the medicare population. J Am Soc Nephrol. 2013;24(9):1478-1483.

23. Lentine KL, Xiao H, Machnicki G, Gheorghian A, Schnitzler MA Renal function and healthcare costs in patients with polycystic kidney disease. Clin J Am Soc Nephrol. 2010;5:1471-1479.

24. Ministero della Salute. Documento di indirizzo per la malattia renale cronica. November; 2014. Available from: http://www.salute.gov.it/ portale/documentazione/p6_2_2_1.jsp?lingua=italiano\&id=2244. Accessed February 20, 2017.
ClinicoEconomics and Outcomes Research

\section{Publish your work in this journal}

ClinicoEconomics and Outcomes Research is an international, peerreviewed open-access journal focusing on health technology assessment, pharmacoeconomics and outcomes research in the areas of diagnosis, medical devices, and clinical, surgical and pharmacological intervention. The economic impact of health policy and health systems

\section{Dovepress}

organization also constitute important areas of coverage. The manuscript management system is completely online and includes a very quick and fair peer-review system, which is all easy to use. Visit http://www.dovepress.com/testimonials.php to read real quotes from published authors. 